\title{
$\mathrm{TiAl}$ 基金属间化合物的塑性变形行为 ${ }^{*}$
}

\author{
蒲忠杰祝 东朱 静察其巩邻敦和
}

（治金工业部钢铁研究总院, 北京 100081）

\section{关铝 $\mathrm{TiAl}$ 化合物、塑性变形、 $R$ 效应}

Kawabta 等首次发现, ${ }^{T} i_{43} \mathrm{Al}_{57}$ 单晶化合物的强度, 在某一温度以下表现出随温度上升 而提高的反常效应 ${ }^{[1]}$ (以下称为 $R$ 效应)。而 Lipisitt $^{[2]}$ 和 $\mathrm{Huang}^{[3]}$ 的研究工作指出, 多晶 $\operatorname{TiAl}(46-60 \mathrm{at} \% \mathrm{Al}$ ) 化合物, 届服强度随温度的升高而降低, 与 Kawabata 的单晶实验结果 相反. 但在文献 $[2,3]$ 中, 屈服强度在室温至 $550^{\circ} \mathrm{C}$ 温度区间缺少实验数据, 因此无法断定 多晶 $\mathrm{TiAl}$ 化合物是否具有 $R$ 效应. 另外, 近年研究发现, 元素 $\mathrm{V} 、 \mathrm{Mn} 、 \mathrm{Ga}$ 能显著改善 $\mathrm{TiAl}$ 化合物常温塑性,但这些元素对 TiAl 化合物高温变形行为的影响, 目前还未见报道. 本文将 较为系统地研究 TiAl 基化合物的塑性变形行为.

\section{一、实验方法}

实验用合金为 $\mathrm{Ti}_{51} \mathrm{Al}_{49} 、 \mathrm{Ti}_{50} \mathrm{Al}_{47.5} \mathrm{~V}_{2.5} 、 \mathrm{Ti}_{5_{1}} \mathrm{Al}_{48} \mathrm{Mn}_{1.0}$ 和 $\mathrm{Ti}_{51} \mathrm{Al}_{47.5} \mathrm{Ga}_{1.5}$ 。合金用电弧炉熔 炼. 铸锭经 $1200{ }^{\circ} \mathrm{C}$ 退火 $25 \mathrm{~h}$ 后, 加工成 $\phi 5 \times 8 \mathrm{~mm}$ 的圆形压缩试样, 实验在 INSTRON 试 验机上进行. 为研究应变速率对材料强度的影响, 先在某一基准速率下将材料变形至 $0.3 \%$, 突然增加应变速率得到高速率下的应加应变曲线, 然后将此曲线外推到 $0.2 \%$ 的应变处, 得到 高应变速率下的屈服强度 $\sigma_{0.2}$ 。

\section{二、实 验结果}

显微组织观察表明,四种合金都由等轴状和层片状两种形态的组织构成, 其中等轴状组织 为单相 $r(\mathrm{TiAl})$ 相,层片状组织由板条状的 $r$ 和板条间的 $\alpha_{2}\left(\mathrm{Ti}_{3} \mathrm{Al}\right)$ 薄膜两相构成.

应变速率为 $5 \times 10^{-5} \mathrm{~s}^{-1}$ 时,材料的屈服强度 $\sigma_{0.2}$ 与温度 $T$ 的关系如图 1 所示. 由图 1 可 见, 经 $\mathrm{V} 、 \mathrm{Mn} 、 \mathrm{Ga}$ 合金化后的 $\mathrm{Ti}(\mathrm{AlX})$ 三元化合物, $\sigma_{0.2}$ 与 $T$ 的关系明显可分为三个区域。 在温度低于 $200^{\circ} \mathrm{C}$ 的 I 区内, $\sigma_{0.2}$ 随 $T$ 的开高而降低; 当温度处于 $200-700^{\circ} \mathrm{C}$ 之间的 II 区 时, $\sigma_{0.2}$ 随温度的升高而提高, 表现出 $R$ 效应; 当温度在高于 $700{ }^{\circ} \mathrm{C}$ 的 III 区时, $\sigma_{0.2}$ 随 $T$ 的进 一步升高而降低. 而 $\mathrm{TiAl}$ 二元化合物, $\sigma_{0.2}$ 随 $T$ 的升高而下降.

图 2 为应变速率敏感系数 $S$ 与温度 $T$ 的关系, $S=\frac{1}{T}\left(\frac{\partial \ln \sigma}{\partial \dot{\varepsilon}}\right)_{T}$. 由图 2 可见, $S$ 随 $T$ 的 增加先减后增. 当温度低于 $400^{\circ} \mathrm{C}$ 时, $S$ 随 $T$ 的上升而下降; 当温度超过 $400{ }^{\circ} \mathrm{C}$ 时, $S$ 则随 $T$ 的上升而增加. 在 $400-500^{\circ} \mathrm{C}$ 之间, $S$ 值较低,有的合金甚至为零. 当温度高于 $700^{\circ} \mathrm{C}$ 时, $S$

本文 1989 年 10 月 5 日收到.

*国家自然科学基金资助项目.

第 6 期 


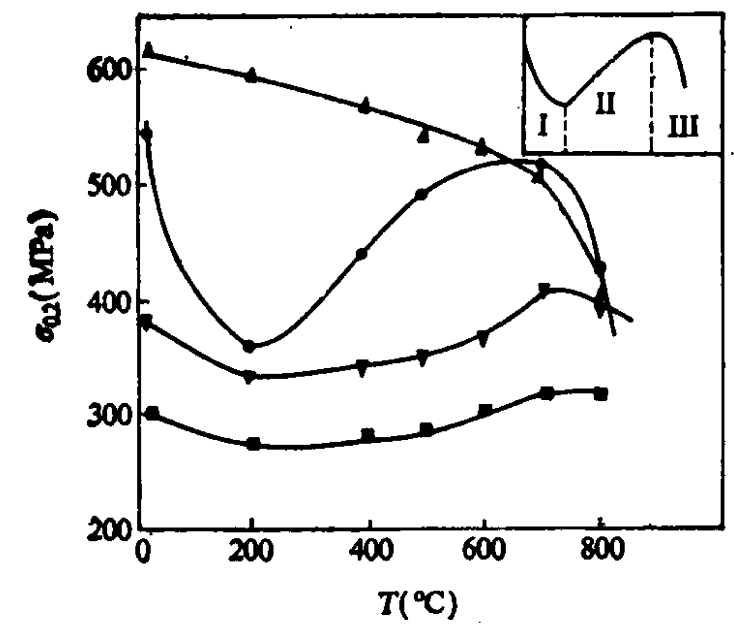

图 1 屈服强度 $\sigma_{\mathrm{c}, 2}$ 与温度 $T$ 的关系

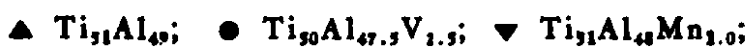 - $\mathrm{Ti}_{1,1} \mathrm{Al}_{37,}, \mathrm{Ga}_{1,9}$

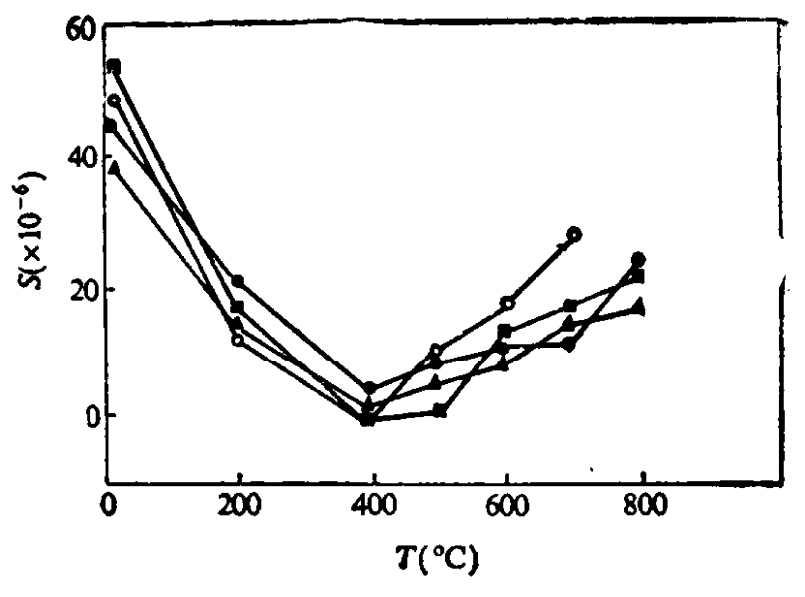

图 2 应变速率敏感系数 $S$ 与温度 $T$ 的关系

随 $T$ 的进一步升高而迅速增加.

\section{三、讨 论}

对于 $\operatorname{Ti}(\mathrm{AlX})$ 三元合金, 在图 1 的 $\sigma_{0.2}-T$ 曲线中发现存在区域 I. 这一发现，尚未见报 道. 将材料在 $25^{\circ} \mathrm{C}$ 变形时的热激活体积 $V^{*}-K T\left(\frac{\partial \ln \dot{\varepsilon}}{\partial \sigma}\right)_{T}$ 作为其应变 量 $\varepsilon$ 的函数（图 3), 由图 3 明显可见:（1）所有合金在 $25^{\circ} \mathrm{C}$ 时 $V^{*}$ 都处于 $\left(1-10^{2}\right) b^{3}$ 之间；(2) $V^{*}$ 不随 $\varepsilon$ 的变化而变化. 这说明合金变形的热激活过程与材料的位错密度和位 错组 态无关, 而与 Pierles 机制一致,因此可认为控制 TiAl 基合金在 I 区内变形的机制为 Pierles 机制 ${ }^{[4]}$.

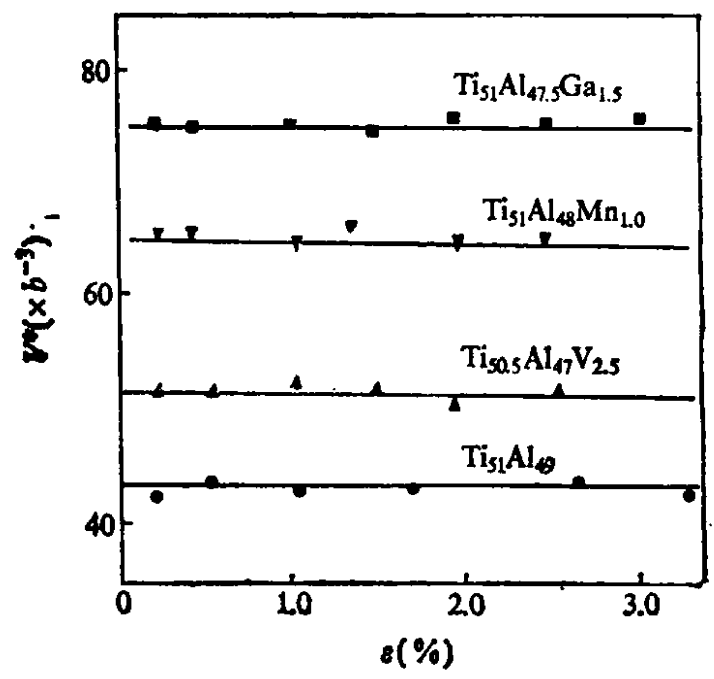

图 3 热擞活体积 $V^{*}$ 与真应变量 8 的关系

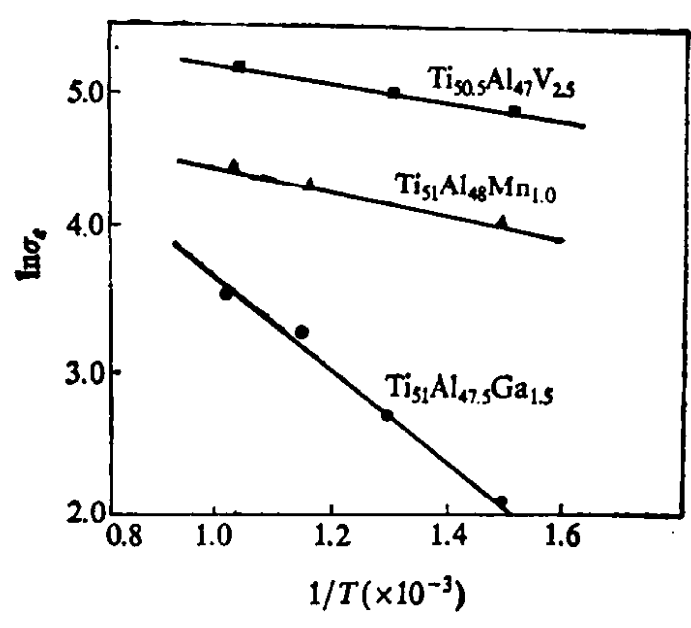

图 $4 \ln \sigma_{s}$ 与 $1 / T$ 的关系

在 $\mathrm{L} 1_{2}$ 型化合物中 $R$ 效应的原因可用 Kear-Wildorf 模型得到解释; 但在 $\mathrm{L} 1_{0}$ 型 $\mathrm{TiAl}$ 化 合物中 $R$ 效应的原因至今还不清楚. Hug ${ }^{[3]}$ 认为 $\mathrm{TiAl}$ 中造成 $R$ 效应的原因和 $\mathrm{L}_{2}$ 型化合物 一样, 仍可用 Kear-Wildorf 模型解释, 而 Greenberg ${ }^{[6]}$ 则认为是由于存在于相互交叉的 $\{111\}$ 面上超偏位错反应形成障碍位错的结果。无论哪种机制, $R$ 效应总是在热激活作用下 
由障碍位错形成的结果. 因此可将温度开高造成的强度增加量 $\sigma_{e}$ 写成如下的形式:

$$
\sigma_{c}=A \exp (-V / K T) \text {, }
$$

其中 $V$ 为障碍位错形成的激活能, $A$ 为常数. 而 $\sigma_{0.2}$ 则可以认为是两部分叠加的结果, 即

$$
s_{0.2}=s_{p}+s_{e} \text {, }
$$

其中 $\sigma_{p}$ 为派纳应力. 显然 $\frac{\partial \sigma_{\ell}}{\partial T} \leqslant 0, \frac{\partial \sigma_{e}}{\partial T}>0$, 因此 $\frac{\partial \sigma_{c, 2}}{\partial T}$ 的正负号取决 于 $\left|\frac{\partial \sigma_{g}}{\partial T}\right|$ 和 $\left|\frac{\partial \sigma_{g}}{\partial T}\right|$ 的大小. 前者大于后者, $\frac{\partial \sigma_{0.2}}{\partial T}<0$, 对应于图 1 中的 $\mathrm{I}$ 区; 前者小于后者, $\frac{\partial \sigma_{0.2}}{\partial T}>0$, 对应于 图 1 中的 II 区. 如果进一步假设 $\sigma_{p}$ 在温度高于 $200^{\circ} \mathrm{C}$ 时不再降低, $\sigma_{e}$ 在温度低于 $200^{\circ} \mathrm{C}$ 时 可以忽略, 则可得到

$$
\sigma_{e}-\sigma(T)-\sigma\left(200^{\circ} \mathrm{C}\right)-A \exp (-V / K T) .
$$

$\ln \sigma_{c}$ 与 $1 / T$ 的关系见图 4 , 由图 4 可见 $\ln \sigma_{c}$ 与 $1 / T$ 成直线关系, 说明式(3) 是合理的.

由图 1 可知,多晶 TiAl 化合物的强度随温度的升高而下降, 与 Lipisitt 和 Huang 的结 果一致. 由图 2 可知, $\mathrm{TiAl}$ 和 $\mathrm{Ti}(\mathrm{AlX})$ 化合物的 $S-T$ 曲线相似, 说明两者的变形机制相同. 为什么 $\mathrm{TiAl}$ 化合物不与 $\mathrm{Ti}(\mathrm{AlX})$ 三元化合物一样表现出 $R$ 效应？可能是 $\mathrm{TiAl}$ 化合物 $\sigma$ ，较 高, 并可维持到更高温度而不降低. 当温度进一步提高时, $\sigma_{p}$ 随温度提高又迅速下降, 尽管 $\sigma_{e}$ 可能随温度升高而提高,但总的效应仍是 $\sigma_{0.2}$ 随温度提高而下降.

\section{四、结 论}

多晶 $\operatorname{Ti}(\mathrm{AlX})$ 三元化合物, 强度与温度的关系中存在三个区域，I区中变形由派纳机制控 制; II 区中强度随温度升高而增加, 其增加量可用 $\sigma_{e}=A \exp (-V / K T)$ 来描述. 而 $\mathrm{TiAl}=$ 元化合物的屈服强度随温度上升而降低, 不表现出 $R$ 效应, 这与 Lipisitt 和 Huang 的结果一 致.

\section{参考文郝}

[1] Kawabate, T., Kanai, T. and Izumi, O., Acta Metall., 33(1985), 1355.

[2] Lipisitt, H. A., Shechtman, D. and Schafvie, R. E., Metall. Trans., 6A(1975), 199.

[3] Huang, S. C., Scripza Metall., 22(1988), 1885.

[4] Sinha, T. K. et al., J. Mat. Sci., 19(1984), 1446.

[5] Hug, G., Loiseau, A. and Veyssiere, P., Phil. Mag., A 57(1988), 499.

[6] Greenberg, B. A. and Gornostirev, Y. U. N., Scripta Metall., 16(1992), 15. 